\title{
The application of OpenCL to accelerate the lossless image compression algorithm based on cascading fragmentation and pixels sequence ordering
}

\author{
A. Khokhlachev ${ }^{1}$, V. Smirnov ${ }^{1}$, A. Korobeynikov ${ }^{1}$ \\ ${ }^{\prime}$ Kalashnikov Izhevsk State Technical University, Studencheskaya 7, 426069, Izhevsk, Russia
}

\begin{abstract}
The previous papers of the authors offer approach to building the ordered sequence of image pixels at lossless compression, which comprises methods of cascading fragmentation and the use of bypasses code book. For fragment sized $6^{*} 6$ the code book contains 22144 various bypasses, the cost of coding to be estimated for every one of them. The search of optimal bypass is an exhaustive search type. The present paper describes ways of increasing the image lossless compression rate by using parallel computation based on OpenCL. Algorithm functions with great runtime were changed in order to transfer calculations to OpenCL using GPU/CPU. The acceleration degree for different algorithm functions gained in experiments amounted to $3 . .32$.
\end{abstract}

Keywords: lossless image compression; cascading fragmentation; pixels sequence ordering; optimal bypass; code book; computational acceleration; parallel computing; open computing language (OpenCL); graphics processing unit (GPU); central processing unit (CPU); Haar integral-valued wavelet transformation; interchannel decorrelation

\section{Introduction}

At the moment there exist both a large number of compression algorithms of particular data classes and universal compression algorithms. This work will address the lossless image compression algorithm based on optimization of bypass image being developed by the authors and described in [1...3]. When processing test images [7], the algorithm gives the average compression ratio of 1.54, which matches the analogues [5]. Let us consider test results by groups of images: 1) in group «2.1.*.tiff» by 1.4262 ) in group «2.2.*.tiff» by 1.5473 ) in group «4.1.*.tiff» by 1.6224 ) in group «4.2.*.tiff» by 1.522 [5]. In addition, the algorithm has some other advantages [5].

To achieve a high compression ratio it is necessary to use a number of demanding algorithm functions, which leads to longer image processing program runtime. Presently, parallel computing is there. The aim of this work is to apply OpenCL to speed up the lossless compression algorithm. To achieve this goal it is necessary to: analyze duration of program execution; find the algorithm functions with time-consuming calculations; consider transfer of these functions to GPU. Image processing performed in the algorithm is based on handling particular fragments, therefore, in general case, such tasks can be carried out simultaneously. Furthermore, it is possible to perform the preprocessing functions for image fragments in parallel as well.

\section{Basic algorithm}

The basic algorithm inherently consists in cascading fragmentation of image [1], the search of the fragment optimal bypass (path) [2], and dynamic programming of pixels delta-code at fragment bypass [6]. After encoding, the obtained data is further compressed by Deflate algorithm using standard libraries. The compression ratio depends on the class of the image being compressed, and on average equals 1.54 for the array of test images [5].

The runtime of image compression program depends on the processed image size. Due to a number of algorithmic solutions such as cascading fragmentation, and the use of bypasses codebook instead of calculating the possible bypasses for each image fragment, the runtime was reduced. However, the image compression duration is still high enough [5]: 1) in group «2.1.*tiff» 101 seconds 2) in group «2.2.*tiff» - 404 seconds 3 ) in group «4.1.*tiff» -24 seconds 4) in group «4.2.*tiff» - 141 seconds.

In computational terms the most complex of the basic algorithm functions is to estimate the encoding cost for all possible bypasses. Meanwhile, this algorithm function is suitable for parallelization, since the optimal bypass choice uses exhaustive search of obtained cost estimates. For a fragment sized $6^{*} 6$ the total bypasses number from the upper left corner is 22144 .

To use all multi-core CPU resources it is necessary to effectively implement paralleling of functions between all cores. The basic program features parallel execution of optimal fragment bypass search cycle done with. Net Framework standard classes (SSE instructions). It is possible to use a more powerful CPU, but even in this case, the speed increase will not be significant.

In recent years the increasing number of programs with parallel data processing use GPU computing [7]. This is dictated by a growing gap in performance between CPU and GPU.

Taking into account the above said, it was decided to move part of the compression algorithm functions to GPU. Obviously, this will require some significant changes in the functions, but it will allow for significant decrease in the program runtime without changing the basic algorithm.

Currently there are several approaches to programs execution on GPU. OpenCL is an open standard [8], which can execute programs on both CPU and GPU of different manufacturers. Therefore, in this research, to speed the algorithm, OpenCL was chosen. 
Image Processing, Geoinformation Technology and Information Security / A. Khokhlachev, V. Smirnov, A. Korobeynikov

At the moment there exist quite a big number of various compression algorithms in general and algorithms for images in particular. Images compressed both as lossy and lossless are widely and effectively used. For example, lossless compression is used in PNG files where the actual compression is implemented with Deflate algorithm [9, 10], which is a combination of LZ and Huffman algorithms in its turn. There are no free turn-key programs available for lossless image compression making use of OpenCL. WinZip is an example of the lossless compression program based on universal algorithm and using OpenCL, which provides for performance increase of about $45 \%$ [11].

In addition to the basic algorithm, image preprocessing was implemented which was described in the authors' previous works: interchannel decorrelation of image color layers [12] and the transformation of pixels matrix based on integer-valued Haar wavelets [13]. These functions can be easily threaded for the implementation on OpenCL.
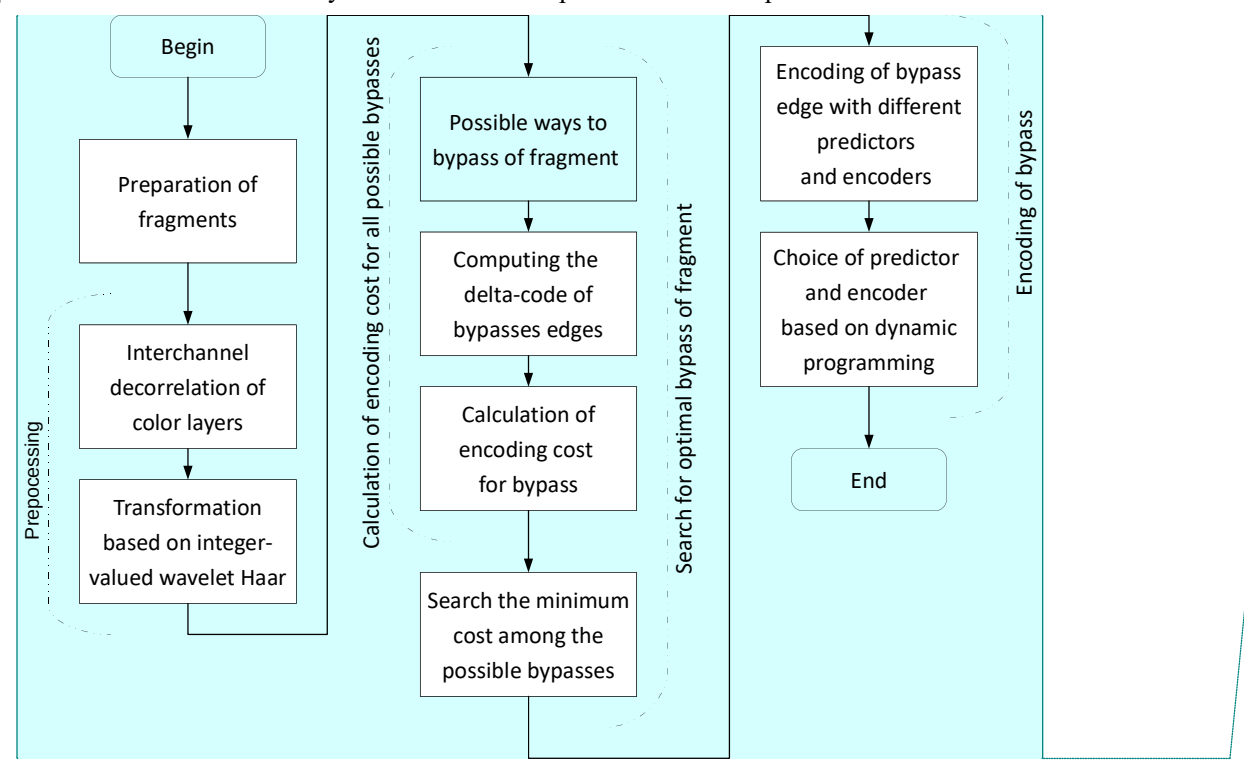

Fig. 1. Lossless image compression algorithm.

Примечание [K1]: По рисунку: preprocessing (не хватает буквы), Haar integer-valued wavelet (порядок слов), to bypass fragments (не нужен предлог of), search of (предлог нужен))),

\section{Methods of acceleration}

The general problem solved in present research is changing the compression software in order to transfer part of calculations to OpenCL. Image compression algorithm is shown in Fig. 1.

\subsection{Preparation of fragments}

The function receives separate color layers of an image. The function output is arrays of separate fragments of fixed size. Pixel values of the fragment nodes beyond the image borders are virtual pixels and the values of these pixels are set as constant (white pixels on Fig. 2). The top left pixels of each fragment on level 0 constitute the fragments on level 1 and so on, as long as the fragments number on a level is more than one. Data structure passed to the OpenCL kernel represents the matrix of image values, the output structure is the array of separate fragments [1].

\subsection{Preprocessing}

\subsubsection{Interchannel decorrelation of color layers}

This function is designed to calculate the interchannel decorrelation between the groups of color channels (layers) of the original image and to find the best variant to group them [12].

When function is started the arrays containing pixels values of all color channels of the fragment, and also the number of channels have to be conveyed (Fig. 3). In addition, data on the possible grouping of channels is needed.

Formula for calculating interchannel decorrelation for arbitrary channels number based on the mean and interchannel differences is applied [12]:

$$
\begin{gathered}
P^{1}=\operatorname{Round}\left(\frac{\sum_{i=1}^{n} X^{i}}{n}\right) \\
P^{i}=X^{1}-X^{i}, i=\frac{1}{2, n}
\end{gathered}
$$


Image Processing, Geoinformation Technology and Information Security/ A. Khokhlachev, V. Smirnov, A. Korobeynikov

where Round is the rounding operation to the nearest integer; $X^{i}$ - pixels value on each of the channels; $k$ - channel index; $n-$ the number of processed channels.
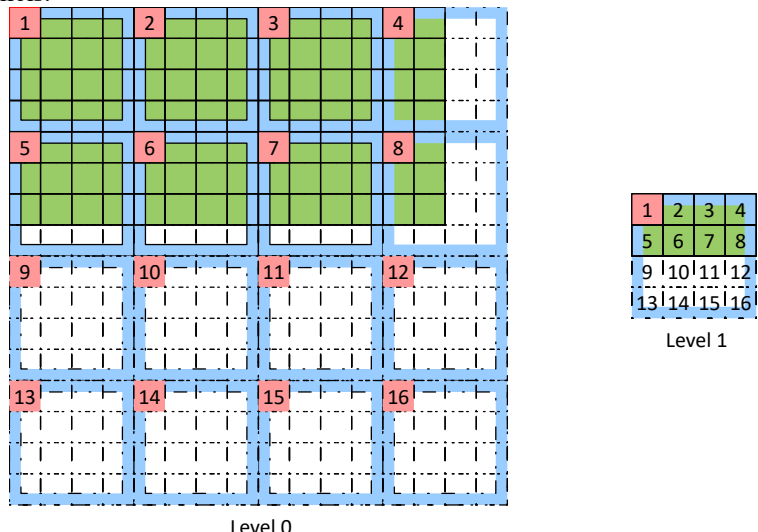

Fig. 2. Preparation of fragments.

The color channels can be independent from each other, therefore, the grouping variant with a minimum encoding costs estimate has to be selected. It is necessary to implement the decorrelation formulas for all dependent channels groups. The minimum channels number in the group is 2 . If the image consists of 3 channels ( 24 bits per pixel), we get the following grouping variants:

$$
\left(X^{1} X^{2} X^{3}\right) X^{1}\left(X^{2} X^{3}\right) X^{2}\left(X^{1} X^{3}\right) X^{3}\left(X^{1} X^{2}\right) X^{1} X^{2} X^{3}
$$

where decorrelation formulas are to be applied to groups of channels in parenthesis

The calculation of decorrelation is performed for all possible groups $(g=1 . . G)$. The result is the index of $g$ grouping:

$$
g=\underset{g}{\operatorname{argmin}}\left(\sum_{g=1}^{G}\left(\sum_{i=1}^{n} \sum_{j=1}^{k} \operatorname{Cost}\left(P^{i_{j}}\right)\right)\right)
$$

where $P_{j}^{i}$ - is the pixel value after the interchannel decorrelation for the grouping index $g ; i-$ channel index; $j$ - pixel index; $n$-number of channels; $k$ - number of pixels number-in the fragment.

Cost is the a seme estimation-function of encoding costs estimate, for example, the length of the Fibonacci code which encoding the value $P_{j_{-}}^{i}$ value, or the estimated length of binary coding:

$$
\log _{2}\left|P^{i_{j}}+1\right|+1
$$

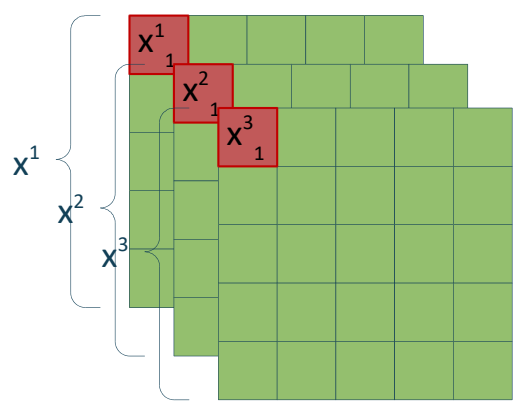

Fig. 3. Interchannel_decorrelation of color layers.

3.2.2. Transformation based on Haar integer-valued wavelets Haar

This function is designed intended for cascading processing of each image fragment by applying an integer-valued version of the Haar wavelet transform [13]

The function is passedreceives an array containing a single channel of image and the number of the processed fragments in by width and height-which need to processed.

In the course of the algorithm execution it uses-additional arrays for each executable OpenCL kernel with athe size equal toof one fragment each are used, for storing the intermediate-interim results of the cascading conversion. 
Image Processing, Geoinformation Technology and Information Security / A. Khokhlachev, V. Smirnov, A. Korobeynikov

The function result isoutputs an array of the size equal to of the original image.

Image matrix should -has to be divided into blocks ef sized $2 * 2$. Then ealeulated the values for $a, h, v, d$ are to be found by the formula [13]:

$$
\begin{gathered}
c_{2}=x_{1}-x_{2} \\
c_{3}=x_{1}-x_{3} \\
c_{4}=x_{1}-x_{4} \\
c_{1}=x_{1}-\operatorname{Round}\left(\frac{1}{4} \sum_{i=2}^{4} c_{i}\right)=x_{1}-z_{1} \approx \frac{1}{4} \sum_{i=1}^{4} x_{i} \\
\quad a=c_{1} \\
h=-\operatorname{Round}(d / 2)+c_{3} \\
v=-\operatorname{Round}(d / 2)+c_{2} \\
d=c_{3}-c_{4}+c_{2}
\end{gathered}
$$

WWhere Round is the rounding operation to the nearest integer; $x_{i}$ - original pixels of the block.

The obtained values of $\mathrm{a}, \mathrm{h}, \mathrm{v}, \mathrm{d}$ should have to be recorded in positions of the matrix- as shown in Fig. 4. Calculation should be carried out as multiresolution at multiple scale, by repeating the transform on the blocks consisting of grouped values $a^{i}$, each time and reducing their size in 2 timesby half for-in each coordinate every time, as long as it is possible to form $2 * 2$ block from $a^{i}$ values on a subsequent scale. Cascading transform will stop then the block $a^{i}$ with size $2 * 2$ is absent.

It should be noted that when-with the fragment sized of $2^{m} * 2^{m}$ it is possible to use preprocessing (interchannel decorrelation, and Haar transform) after the function of dividing on the-fragmentations. In this case, the Haar transformation is possible only within the same fragment. With In this approach, the fragment encoding is completely independently of the other fragments and therefore the decoding is possible for a single fragment.
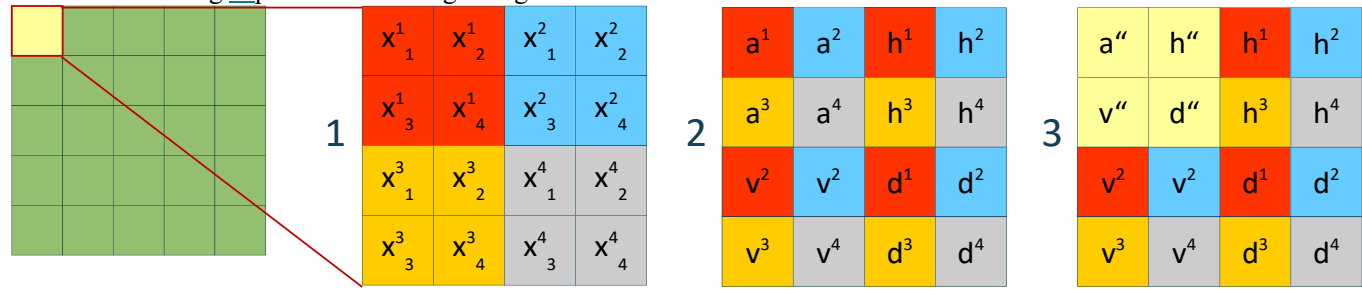

Fig. 4. Multireselution-Multiple-scale transformation based on $\underline{\text { Haar }}$ wavelet Haar.

\subsection{Search for optimal bypass of fragment}

The function receives the fragments obtained after preprocessing functions (integer-valued Haar transform, interchannel decorrelation of color layers).

\subsubsection{Calculation of encoding cost for all possible bypasses of fragment}

\subsubsection{Possible ways to bypass $\theta$ f a fragment}

Encoding and decoding algorithms have iInformation about all the possible bypasses for a given fragment size. is known for encoding and decoding algorithms.

All bypasses (paths) have been calculated in advance and eonstitute-stored in the bypasses codebook [2].

Therefore, in encoding and decoding only need to know the bypass index, but not the edges of bypass (path), is the only prerequisite for encoding and decoding., but not the edges of bypass (path).

\subsubsection{Computing the delta-code of bypasses edges}

This function is designed to calculate the difference of values for all pairs of nodes that make up the edge on a given fragment bypass [2]. In the course of the functionThe algorithm uses the previously prepared fragments.

The result is a list of arrays containing the delta-code of all edges for each fragment.

It is necessaryFor each fragment you need to make-compile an array of differences between the nodes values (delta-code) connecting the edge $e$ is calculated according to thewhich is done with formula:

$$
\Delta_{e}=x_{\text {start }(e)}-x_{\text {stop }(e)}
$$

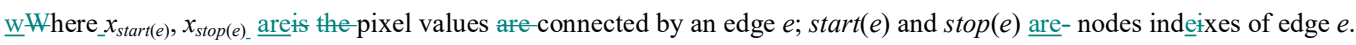

3.3.1.3. Calculation of encoding costs for bypass

For each fragment, estimates-the encoding costs for each of the possible bypasses are calculated. The function receives the previously prepared fragments and details-data onef all the fragment bypasses in afrom the codebook. 
Image Processing, Geoinformation Technology and Information Security / A. Khokhlachev, V. Smirnov, A. Korobeynikov

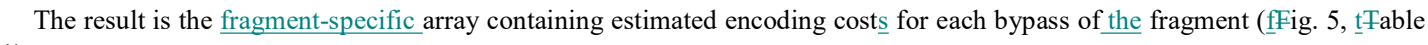
1).

Estimationg of the encoding costs of a bypass through all edges (with its-all delta-codes) of bypass edges it is possible to producecan be done in different ways. The cost of bypass fFor each fragment is needhas to findbe found the cost of the bypass:

$$
\Sigma_{s}=\sum_{e=1}^{E} \operatorname{Cost}\left(\Delta_{e}\right) \cdot z^{e_{s}}
$$

where $E \underline{\text { is }}$ - the length of bypass (the number of edges); $e$ - edge index; $S$ - the number of bypasses; $s$ - bypass index; $\Delta_{e}$ delta-code of edge; $z_{\mathrm{s}}^{e}$ - presence of thean-edge $e$ in bypass $s$; Cost - is the somean estimation function of encoding costs, it is similar to the Cost in interchannel_decorrelation function.

It should be noted that the estimate of bypasses encoding costs based on the table 1 is effective from the point of view of parallel computing. In this function there isrelies on parallel processing of all possible bypasses downloaded from the codebook, for all fragments making up, forming the processed image.

3.3.2. Search of the minimum among the possible bypasses

After the estimates of encoding costs of all paths is selectedare estimated, and the save path of each fragment-bypass with the smallest estimate for each fragment is picked and saved.

$$
s=\underset{s}{\operatorname{argmin}}\left(\Sigma_{s}\right)
$$

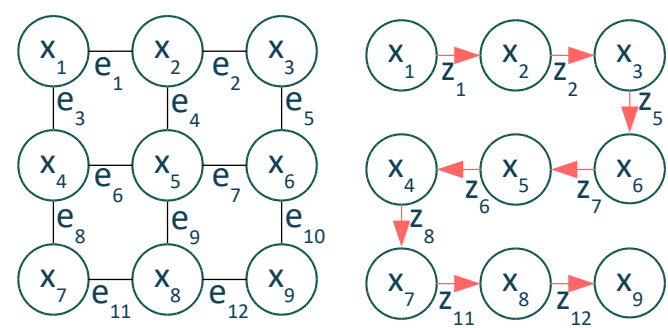

Fig. 5. Example of bypass ofen fragment $3 * 3$ fragment.

Table 1. Calculation of encoding cost for bypass in fragment $3 * 3$ fragment.

\begin{tabular}{|c|c|c|c|c|c|}
\hline $\boldsymbol{e}$ & $\Delta_{\boldsymbol{e}}$ & $\boldsymbol{z}_{1}^{e}$ & $\boldsymbol{z}_{2}^{e}$ & $\cdots$ & $\boldsymbol{z}_{\mathbf{8}}^{e}$ \\
\hline $\mathbf{1}$ & $\Delta_{1}$ & 1 & 1 & $\cdots$ & 0 \\
\hline $\mathbf{2}$ & $\Delta_{2}$ & 1 & 1 & $\ldots$ & 1 \\
\hline $\mathbf{3}$ & $\Delta_{3}$ & 0 & 0 & $\ldots$ & 1 \\
\hline $\mathbf{4}$ & $\Delta_{4}$ & 0 & 0 & $\ldots$ & 1 \\
\hline $\mathbf{5}$ & $\Delta_{5}$ & 1 & 1 & $\ldots$ & 1 \\
\hline $\mathbf{6}$ & $\Delta_{6}$ & 1 & 1 & $\ldots$ & 0 \\
\hline $\mathbf{7}$ & $\Delta_{7}$ & 1 & 0 & $\ldots$ & 0 \\
\hline $\mathbf{8}$ & $\Delta_{8}$ & 1 & 1 & $\cdots$ & 1 \\
\hline $\mathbf{9}$ & $\Delta_{9}$ & 0 & 1 & $\cdots$ & 1 \\
\hline $\mathbf{1 0}$ & $\Delta_{10}$ & 0 & 1 & $\cdots$ & 1 \\
\hline $\mathbf{1 1}$ & $\Delta_{11}$ & 1 & 0 & $\cdots$ & 1 \\
\hline $\mathbf{1 2}$ & $\Delta_{12}$ & 1 & 1 & $\cdots$ & 0 \\
\hline
\end{tabular}

\subsection{Encoding of bypass}

This function is designed to encode the previously found optimal bypass. That is, the obtained array of bypass nodes values

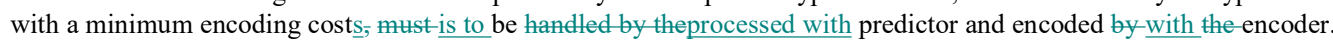

It should be nNoted that the-encoding of bypass which-previously was-found as optimal bypass-can be performed in various ways. In particular, there can be usedcertain known generiecommon methods can be used: Huffman algorithm or arithmetic coding.

As for the consideredIn suggested algorithm it is offered to perform the algorithm the bypass encoding of bypassis suggested which employs -using a more sophisticated method [6]: 1) using a set of predictors and encoders for-to encode the bypass edge; 2) using dynamic programming for choice ofto choose predictors and encoders for edge in purpose to optimize (to-minimize) $\theta$ f the total bypass encoding costs-of bypass. 
Image Processing, Geoinformation Technology and Information Security / A. Khokhlachev, V. Smirnov, A. Korobeynikov 1.1.1.3.4.1. Encoding of bypass edge with different predictors and encoders

In the simplest-most common case, the edge delta-code described above is used as the-a predictor-uses the edge delta-code deseribed above, and Fibonacci codes are used as encoders-uses the Fibonacei codes-[3]. In this case, the applicationying of dynamic programming to select the predictor and the encoder is not required. In a-more complex cases the number $\theta$ f choices-of predictors and encoders variants may be more than one. For example, it is possible case with the predictors are possible not only on the basis of not only the finite difference of the first degree, but higher degrees, and Rice codes with different bases can be used as encoderswith the coders with use Rice codes with different bases. The use of a set of predictors and a set of encoders increases the resulting image compression ratio, but this raises the problem of choosing the best predictor and encoder for the current section of the bypass array.

\subsection{Choice of predictor and encoder based on dynamic programming}

In this embodiment, This variation of compression algorithm to encode-with which each bypass edge is encoded uses the most optimal encoding parameters (predictor/encoder) based ondefined by dynamic programming [6]. In start of the-When function is started, it is loaded the table of encoding cost for every encoder for values of every predictors for all pixels on edges of the optimal bypass is downloaded and executed.

In the result $t$ The function ereates-produces a data file containing information with on the size of the-encoded using enceders predictors values for edges-and additional information - optimal switching of the predictors/encoders for edges of bypass encoding [6].

Due to the complexity of the dynamic programming algorithm it was found possible to transfer toto run on OpenCL managed to transfer-only a small part- responsible for the coding directly to OpenCL. This part contains branching, and is switching large sections of the algorithm takes place. These operations are an integral part of the algorithm, or changand changinge the calculations flow in aim of parallel execution-without the-use of branches is not possible.

\subsection{Results and Discussion}

Sereen form The interface of the developed software is shown in Fig. 6. The program displays the following information: used hardware processor deviceprocessing unit and software platform being used; the number of files to be processed; the current processed file; the execution duration-time of the compression program particular functions; the execution duration ofoverall compression time; the compression ratio.

To useHardware requirements for-OpenCL acceleration requires the presence ofare GPU or CPU with support of OpenCL 1.2 [8]. You must install tThe appropriate OpenCL support software which-distributed with equipment is to be installed.

To-The compilatione of the developed image compression program requires the following software components [8]: 1) DotNetZip library, for the final compression of results using thewith -Deflate algorithm; 2)) to provide OpenCl bindings for CH, use the Cloo library from OpenTk to link OpenCL to C\#; 3) to compile and execute kernels on the GPU you must have-the required header files for OpenCL support in order to compile and execute kernels on the GPU. Ionic.Zip.dll library is used tFo compress the encoding results-used library Ionic.Zip.dll. In addition is usedOther requirements include a set of libraries to support work OpenCL running, bindings these libraries to .Net Framework and the source codes of the OpenCL kernels compiled in course of program execution.

The basic program required about $250 \mathrm{MB}$ of RAM. When algerithm was To adapted the algorithm for accelerating on OpenCL was added usinglarge-volume arrays were addedef large volume and hence memory required-demand increased to 900 $\mathrm{MB}$.

\begin{tabular}{|c|c|}
\hline 啰 Image compression & \begin{tabular}{|l|l|l|} 
& 回 & $x$ \\
\end{tabular} \\
\hline$\nabla$ Use OpenCL & \multirow{9}{*}{ 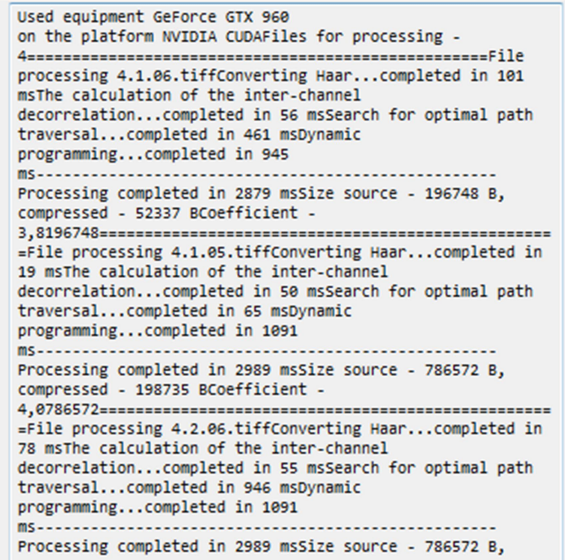 } \\
\hline NVIDIIACUDA & \\
\hline Devices & \\
\hline \multirow{5}{*}{$\nabla$ GeForce GTX 960} & \\
\hline & \\
\hline & \\
\hline & \\
\hline & \\
\hline & \\
\hline File path & C:Users Ivssmimov. DEV Pictures lcompress test2 \\
\hline
\end{tabular}

Отформатировано: многоуровневый + Уровень: $3+$ Стиль нумерации: $1,2,3, \ldots+$ Начать с: $1+$ Выравнивание: слева + Выровнять по: 0 см + Отступ: 0 см

Отформатировано:
многоуровневый + Уровень: $3+$
Стиль нумерации: $1,2,3, \ldots+$ Начать
с: 1 + Выравнивание: слева +
Выровнять по: 0 см + Отступ: 0 см

Выровнять по: 0 см + Отступ: 0 см
Отформатировано:

многоуровневый + Уровень: $1+$ Стиль нумерации: $1,2,3, \ldots+$ Начать с: $1+$ Выравнивание: слева + Выровнять по: 0 см + Отступ: 0 см 
Image Processing, Geoinformation Technology and Information Security / A. Khokhlachev, V. Smirnov, A. Korobeynikov

Fig. 6. Sereen formInterface of the developed software.

Test batch-sample of images designed-is intended to assess acceleration of all core functions of modified program in eomparing withrelative to basic one. To estimate the dependence of the program speed-runtime on the toimages size, the bateh have the-images of different sizes are sampled. To check usedFor test purposes 4 images from the standard set provided by the Institute of signal processing and images processing were used: 4.1.06.tiff, 4.2.05.tiff, 4.2.06.tiff, 4.2.07.tiff. The color depth of the images are-is 24 bit. Image sizes are: $256 * 256,512 * 512,1024 * 1024,2048 * 2048$.

At tFesting was performed using image compression was performed with bothas- the the basic program on the CPU AMD Phenom II X4 955 platform and a program using OpenCL. For testing OpenCL parallel processing was used different 4 different devices were used: 1) GPU AMD Radeon HD6850; 2) GPU Nvidia GTX 960; 3) CPU AMD Phenom II X4 955; 4) CPU AMD FX-4300. The time spent on the particular functions of the algorithm, and the total processing time for each image are given in Table 2 and Fig. 7, where F1 is - integer-valued Haar transform, F2 - interchannel_decorrelation of color layers, F3 - search of the optimal bypass, $\mathrm{F} 4$ - encoding bypass using dynamic programming.

When testing for $\mathrm{eE}$ Each fragment hadwas fixed size: $6^{*} 6$ pixels, and the number of bypasses: 22144 at testing.

It should be noted, that when using thewith -GPU Nvidia GTX 960 configuration, according to Profiler, the load does not exceed $60 \%$ while-despite numerous sthe high number of processing devices and high work frequency, , according to Profiler the load does not exceed 6 Compression-The image of size 2048*2048 pixels size could not be compressed on the GPU AMD Radeon HD6850 failed to produce due to the lack ofinsufficient graphics memory. In the future, to avoid this situationsuch failures, the necessary modification of the program needs to be modified: to run the performed calculations flow should be divided into several groups-threads and processed sequentially.

In the basic program were not implementedthe functions of the integer-valued Haar transform and the-interchannel decorrelation were not implemented, and therefore, testing of these functions was not carried out.

Testing showed-yielded approximately the same reduction in the compression total overall compression time when usingwith both CPU and GPU application. The larger the size of the processed image, the greater the acceleration obtained as long as there is memory available to for OpenCL.

GPU showed the best resultsperformed better in the-for searching of the optimal bypass task. CPU well with the funetion of handles dynamic programming well; due to because of presences of a large number of branches in the function, despite the small number $\theta$ f processerof processor cores.

Time spent on cEalculatingens of interchannel_decorrelation and integer-valued Haar transform is performed-using OpenCL for a shert timeis insignificant compared to total compression time.

Table 2. Results of processing of test images.

\begin{tabular}{|c|c|c|c|c|c|c|c|c|c|c|}
\hline \multirow{2}{*}{\multicolumn{2}{|c|}{$\begin{array}{l}\text { Used } \\
\text { program / } \\
\text { device }\end{array}$}} & \multirow{3}{*}{$\begin{array}{l}\text { Image } \\
\text { size, } \\
\text { pixels } \\
256 * 256\end{array}$} & \multicolumn{5}{|c|}{$\begin{array}{l}\text { Execution time of compression } \\
\text { particular functions, miliseconds }\end{array}$} & \multicolumn{3}{|c|}{$\begin{array}{l}\text { Acceleration, } \\
\text { Times }\end{array}$} \\
\hline & & & F1 & F2 & F3 & F4 & Total & F3 & F4 & Total \\
\hline \multirow{16}{*}{ 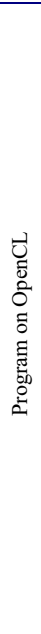 } & \multirow{4}{*}{$\begin{array}{l}\text { AMD } \\
\text { Radeon } \\
\text { HD } 6850\end{array}$} & & 71 & 65 & 677 & 528 & 2581 & 2,2 & 9,0 & 2,8 \\
\hline & & $512 * 512$ & 43 & 63 & 913 & 1129 & 4643 & 6,1 & 13,4 & 5,2 \\
\hline & & $1024 * 1024$ & 66 & 187 & 2017 & 4492 & 15217 & 12,9 & 13,5 & 6,4 \\
\hline & & $2048 * 2048$ & 151 & 438 & - & - & - & - & - & - \\
\hline & \multirow{4}{*}{$\begin{array}{l}\text { Nvidia } \\
\text { GeForce } \\
\text { GTX } 960\end{array}$} & $256 * 256$ & 23 & 20 & 160 & 360 & 2057 & 9,4 & 13,2 & 3,5 \\
\hline & & $512 * 512$ & 11 & 36 & 411 & 1153 & 4402 & 13,6 & 13,1 & 5,5 \\
\hline & & $1024 * 1024$ & 33 & 148 & 1474 & 4226 & 13416 & 17,7 & 14,4 & 7,3 \\
\hline & & $2048 * 2048$ & 122 & 675 & 5354 & 14530 & 50031 & 31,8 & 15,9 & 8,6 \\
\hline & \multirow{4}{*}{$\begin{array}{l}\text { AMD } \\
\text { FX-4300 }\end{array}$} & $256 * 256$ & 34 & 30 & 350 & 499 & 2581 & 4,3 & 9,5 & 2,8 \\
\hline & & $512 * 512$ & 18 & 48 & 913 & 1146 & 4207 & 6,1 & 13,2 & 5,7 \\
\hline & & $1024 * 1024$ & 45 & 206 & 3324 & 4093 & 15514 & 7,9 & 14,8 & 6,3 \\
\hline & & $2048 * 2048$ & 172 & 920 & 13164 & 13629 & 58567 & 12,9 & 17,0 & 7,4 \\
\hline & \multirow{4}{*}{$\begin{array}{l}\text { AMD } \\
\text { Phenom II } \\
\text { X4 } 955\end{array}$} & $256 * 256$ & 31 & 32 & 455 & 664 & 2407 & 3,3 & 7,2 & 3,0 \\
\hline & & $512 * 512$ & 21 & 56 & 1378 & 1222 & 5981 & 4,0 & 12,4 & 4,0 \\
\hline & & $1024 * 1024$ & 67 & 239 & 4571 & 4477 & 16777 & 5,7 & 13,6 & 5,8 \\
\hline & & $2048 * 2048$ & 227 & 992 & 18432 & 12804 & 61350 & 9,2 & 18,1 & 7,0 \\
\hline \multirow{4}{*}{\multicolumn{2}{|c|}{$\begin{array}{l}\text { Basic program / } \\
\text { AMD Phenom II } \\
\text { X4 } 955\end{array}$}} & $256 * 256$ & - & - & 1504 & 4751 & 7273 & 1,0 & 1,0 & 1,0 \\
\hline & & $512 * 512$ & - & - & 5570 & 15155 & 24183 & 1,0 & 1,0 & 1,0 \\
\hline & & $1024 * 1024$ & - & - & 26107 & 60698 & 97297 & 1,0 & 1,0 & 1,0 \\
\hline & & $2048 * 2048$ & - & - & 170161 & 231398 & 431555 & 1,0 & 1,0 & 1,0 \\
\hline
\end{tabular}


Image Processing, Geoinformation Technology and Information Security / A. Khokhlachev, V. Smirnov, A. Korobeynikov

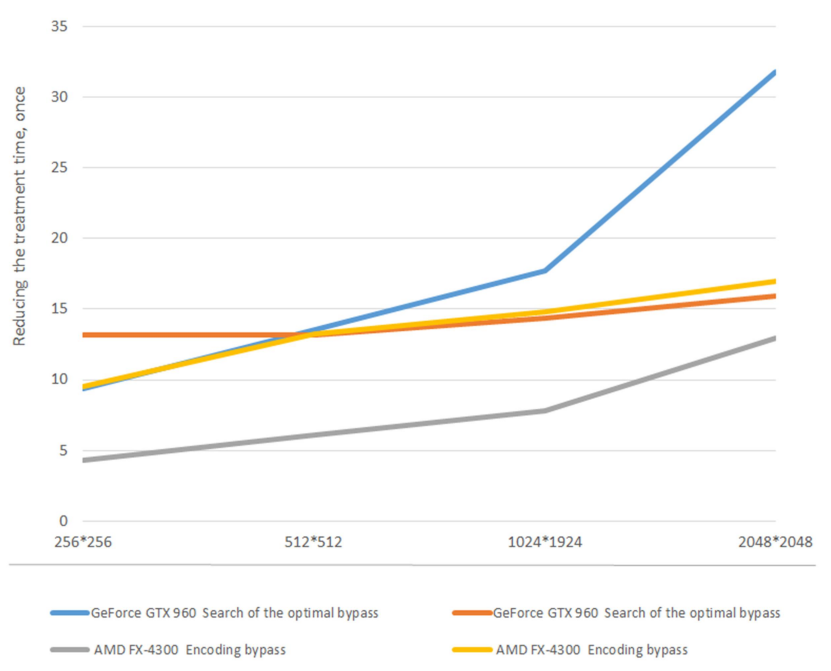

Fig. 7. Comparison of image compression acceleration.

\subsection{Conclusion}

In the course of this work was modified-the basic program for with the aim of increasing shortening its runtimethe speed. The pParallel processing based on OpenCL was used for program acceleration. This solution significantly affected the processing speed, enabling making it possible to reduce computational time. This modification will allow-provide for more efficient use of the program in the future, will-facilitate future-further research aimed at improving the compression ratio.

The changing of optimal bypass search function allowed for to obtain the acceleration up to 32-fold acceleration on the-large images. This acceleration has been achieved because of executing OpenCL-functions executed on OpenCL are almost linear, and branching, even where they arewhen it is the case, is limited tohave only a few simple operations. Furthermore, For future program modification thet acceleratione of this function is important for future program modification because it is -makes possible to use fragments of larger size that which was previously impossibleunattainable earlier due to too muchgreat execution time. Among other things Moreover, fragments with the-sized $\theta f 2^{k} * 2^{k}$ will effectivelyallow -applying the integer-valued Haar transformation for the fragmentto them, and will allow to-compressing every each fragment separately.

Somewhat worse is the situation withAs regards dynamic programming, the prospects are not as bright-during encoding fragment bypass. Speed-Performance managed to increase was gained mostly by due to of some operations, shutdown ofcancel of operations which need onlyused solely for debugging purposes, and the-use of the packet data read operations. The part that runs on OpenCL gives the increase in performance is of only about $30 \%$ compared with-to ordinary parallel computing. On the other hand, even this result is relatively good-enough, given the factprovided that OpenCL function has rathera wide-large enough branching. It should be nNoted that the-bypass encoding can be performed in various ways, for example,e.g. with Huffman algorithm or arithmetic coding.

\section{References}

[1] Smirnov VS, Korobeynikov AV. Cascade Image Splitting into Fragments at Lossless Compression on Basis of Image Bypass Optimization. Bulletin of Kalashnikov ISTU 2012; 2: 143-144.

[2] Korobeynikov AV, Smirnov VS. Optimal Bypass Definition with Code Book Application at Images Lossless Compression. Bulletin of Kalashnikov ISTU 2012; 3: 114-115.

[3] Smirnov VS, Korobeynikov AV. Ordering the numeric sequence of image pixels at lossless compression. I International Forum "Instrumentation Engineering, Electronics and Telecommunications (November, 25-27, 2015, Izhevsk, Russian Federation), 2015; 175-180.

[4] Sample images from the site of University of Southern California. URL: http://sipi.usc.edu/database/database.php?volume=misc (2017-01-10)

[5] Smirnov VS, Korobeynikov AV. The results of testing lossless compression algorithm based on cascade fragmentation method and ordering pixels sequence. II International Forum "Instrumentation Engineering, Electronics and Telecommunications (November, 23-25, 2016, Izhevsk, Russian Federation), 2016.

[6] Korobeynikov AV. The Use of Dynamic Programming and Fibonacci Codes for InterchannelDecorrelation. The Three-Channel Signals Lossless Compression. Bulletin of KIGIT 2010; 1: 72-81. URL: http://elibrary.ru/item.asp?id=18348092 (2017-01-10).

[7] Denny Atkin. Computer Shopper: The Right GPU for You. URL: http://www.computershopper.com/feature/the-right-gpu-for-you (2017-01-10)

[8] Official webpage of the standart OpenCL. URL: https://www.khronos.org/opencl/ (2017-01-10).

[9] Portable Network Graphics (PNG) Specification (Second Edition). URL: https://www.w3.org/TR/PNG/ (2017-01-10)

[10] PNG Home Site. URL: http://www.libpng.org/pub/png/ (2017-01-10).

[11] WinZip official webpage. URL: http://www.winzip.com/win/ru/index.htm (2017-01-10).

[12] Franchenko RS, Korobeynikov AV. InterchannelDecorrelation for Any Number of Channels at Lossless Compression of Multichannel Signals. Bulletin of Kalashnikov ISTU 2010; 1: 87-88. 
Image Processing, Geoinformation Technology and Information Security / A. Khokhlachev, V. Smirnov, A. Korobeynikov

[13] Smirnov VS, Korobeynikov AV. Lossless Image Compression Based On Integral-Valued Haar Wavelets. Intelligent Systems in Manufacturing. Bulletin of Kalashnikov ISTU 2013; 2: 158-160. 